\title{
Sepsis fulminante post esplenectomía. Reporte de un caso y revisión de la literatura.
}

\section{Fulminant sepsis post splenectomy. Report of a case and review of the literature.}

\author{
Luis Novoa-Millones', \\ Karina Butrón-Valencia ${ }^{2}$
}

\author{
Novoa Millones L, Butrón-Valencia K. Sepsis fulminante post \\ esplenectomía. Reporte de un caso y revisión de la literatura. Rev \\ Soc Peru Med Interna. 2020;33(4): I7I-I75. \\ https://doi.org/10.36393/spmi.v33i4.567
}

\begin{abstract}
RESUMEN
Se presenta el caso de un paciente varón de 37 años de edad quien fue admitido en emergencia por presentar lesiones purpúricas generalizadas y antecedente de haber sido esplenectomizado 20 años atrás. Rápidamente progresó a choque distributivo, insuficiencia respiratoria, falla multiorgánica, CID y muerte. El diagnóstico final fue purpura fulminans (PF) secundaria a sepsis fulminante post esplenectomía por bacteriemia debida a Streptococcus pneumoniae. Se revisan los conceptos relacionados y el rol del bazo en la respuesta a infecciones por bacterias encapsuladas.
\end{abstract}

Palabras claves: Sepsis, púrpura fulminante, post esplenectomía, Streptococcus pneumoniae.

\section{ABSTRACT}

This is the case of a 37-year-old male patient who was admitted to emergency ward for widespread purplish lesions and a history of being splenectomized 20 years ago. He quickly progressed to distributive shock, respiratory failure, multiorgan failure, DIC and death.The final diagnosis was purpura fulminans (PF) secondary to fulminant post-splenectomy sepsis by bacteriemia due to Streptococcus pneumoniae. Related concepts and the role of spleen in responding to infections by encapsulated bacteria are reviewed.

Keywords: Sepsis, fulminant purple, post splenectomy, Streptococcus pneumoniae.

\section{INTRODUCCIÓN}

El bazo es el organo linfoide más grande del organismo y la mayor fuente de producción de anticuerpos. Por

I Médico internista. Servicio de Medicina Interna Clínica AUNA Guardia Civil. Profesor de la Facultad de Medicina de la Universidad Peruana San Juan Bautista, Lima, Perú.

2 Médico internista. Servicios de Emergencia de la Clínica Centenario Peruano-Japonesa y del Hospital Central de la Fuerza Aérea del Perú, Lima, Perú. tanto, aquellos pacientes con hipoesplenismo o asplenia anatómica (resección quirúrgica parcial o total del bazo) o funcional (pérdida de la función del bazo) tienen un riesgo mayor de infecciones invasivas severas y morbimortalidad incrementadas a lo largo de la vida. ${ }^{1,2}$ Las causas más frecuentes de asplenia o hipoesplenismo son la esplenectomía quirúrgica después de trauma o por enfermedades hematológicas.,

Se presenta el caso de un paciente esplenectomizado que desarrolla sepsis fatal.

\section{CASO CLÍNICO}

Un paciente varón de 38 años fue admitido en el departamento de emergencia por presentar lesiones purpúricas generalizadas. Sus síntomas comenzaron 24 horas antes con fiebre intermitente de hasta $40^{\circ} \mathrm{C}$, malestar general, poliartralgias y polimialgias. Solicitó evaluación médica y recibió metamizol 1000 mg vía oral condicional a síntomas. Las manifestaciones mejoraron parcialmente con la medicación; sin embargo, 20 horas más tarde presentó "coloración azulada" en cara, manos y tórax además de dolor en manos y pies, por lo cual acudió a emergencia. No presentó disnea, tos ni signos de dificultad respiratoria. $\mathrm{Su}$ 
historia anterior solo reveló esplenectomía 20 años antes por trauma abdominal debido a un accidente de tránsito, sin vacunación posterior.

Al examen físico se observaron lesiones purpúricas de pocos milímetros y rápidamente confluentes en cara, tórax anterior y posterior, y en las cuatro extremidades. Figura 1 (A, B y C).). Frialdad distal, presión arterial 100/70 mm $\mathrm{Hg}$ que rápidamente progresó a hipotensión hasta requerir vasopresores, frecuencia cardiaca $120 / \mathrm{min}$, frecuencia respiratoria $22 / \mathrm{min}$ y $\mathrm{SatO}_{2}$ no detectable por oximetría de pulso.

Exámenes auxiliares: $\mathrm{pH} 7,02 ; \mathrm{PCO}_{2} 33,1 \mathrm{~mm} \mathrm{Hg}$; $\mathrm{PO}_{2} 24,3$ $\mathrm{mm} \mathrm{Hg} ; \mathrm{HCO}_{3} 8,5 \mathrm{mmol} / \mathrm{L}$ y lactato $5,88 \mathrm{mmol} / \mathrm{l} ; \mathrm{SatO}_{2}$ $25 \%$. Hemograma: leucocitos $8,87 \times 10^{3} / \mathrm{mm}^{3}$ (abastonados $1,33 \times 10^{3} / \mathrm{mm}^{3}$, linfocitos $0,53 \times 10^{3} / \mathrm{mm}^{3}$, segmentados 7 $\mathrm{x} 10^{3} / \mathrm{mm}^{3}$ ) y plaquetas $64 \times 10^{3} / \mathrm{mm}^{3}$. INR 5,24 y TP 60,7 ,; fibrinógeno $<180 \mathrm{mg} / \mathrm{dl}$, TTP 143,3" y dímero D 20,5 ug/ ml. Creatinina $5,08 \mathrm{mg} / \mathrm{dl}$ ), bilirrubinas totales $1,96 \mathrm{mg} /$ dl, bilirrubina directa 1,48 mg/dl, TGP $206 \mathrm{U} / 1$, albumina $1,9 \mathrm{~g} / \mathrm{dl}$.

Radiografía de tórax. No evdencia de neumonía.

El paciente fue sometido a intubación orotraqueal (IOT), soporte vasopresor y ventilación mecánica. Se hizo el diagnostico de choque distributivo, falla multiorgánica y coagulación intravascular diseminada (CID). Se inició terapia antibiótica con meropenem y vancomicina, hidrocortisona $100 \mathrm{mg} \mathrm{EV}$ cada 8 horas, plasma fresco congelado y dosis altas de vasopresores. Cuarenta y ocho horas más tarde se aisló Streptococcus pneumoniae sensible a penicilina en dos hemocultivos. A pesar de la terapia agresiva, el paciente falleció tras 72 horas del inicio de sus síntomas. El diagnóstico final fue purpura fulminans (PF) secundaria a sepsis fulminante post esplenectomía debido a Streptococcus pneumoniae.

\section{DISCUSIÓN}

El bazo es el ógano linfoide y el filtro de sangre más grande del cuerpo humano. ${ }^{1,5,6} \mathrm{Su}$ localización anatómica y microestructura lo convierten en un lugar crucial de exposición temprana a bacterias encapsuladas, además de ser una gran fuente de anticuerpos. ${ }^{7}$ Por tanto, la ausencia parcial o total y el hipoeslenismo confieren un riesgo muy alto de desarrollar infecciones graves, principalmente por bacterias encapsuladas, $S$. pneumoniae, $N$. meningitidis y $H$. influenzae tipo b. ${ }^{8}$ Las causas más frecuentes de hipoesplenismo se muestran en la tabla 1.

Este caso refleja muy bien la presentación clínica y evolución rápidamente progresivas de sepsis fulminante postesplenectomía, o por sus siglas en inglés OPSI (Overwhelming Post-Splenectomy Infection), en un paciente esplenectomizado muchos años antes que se complica con púrpura fulminas $(\mathrm{PF})$ y termina en exitus fatalis a los tres días de iniciado el cuadro.

Existe una variedad de conceptos alrededor de las infecciones por encapsulados y de forma particular por Streptococcus pneumoniae. Estos se resumen mejor en la tabla 2.

\section{Infección neumocócica invasiva}

Los pacientes sin bazo tienen 12-25 veces más probabilidad de tener enfermedad neumocócica invasiva (IPD, por sus siglas en inglés, Invasive Pneumococcal Disease) que la población general. ${ }^{12}$ Además, el riesgo de muerte por sepsis es 200 veces mayor comparado con aquellos que tienen función esplénica normal. ${ }^{13} \mathrm{La}$ IPD se define como la infección confirmada por aislamiento de Streptococcus pneumoniae de un sitio normalmente estéril (ej. sangre, líquido cefalorraquídeo, endocardio). ${ }^{14}$ De esta forma puede causar meningitis, endocarditis, neumonía y, con mucha menor frecuencia, peritonitis, artritis séptica $u$ osteomielitis. ${ }^{15}$ Los tres primeros pueden ser potencialmente fatales con tasas de mortalidad de hasta 31\%. ${ }^{16}$ Los principales factores de riesgo para IPD son extremos de la vida ( $<2$ años o $\geq 65$ años), enfermedad cardiovascular crónica, enfermedad pulmonar crónica, enfermedad renal crónica, cirrosis hepática, asplenia funcional o anatómica, diabetes mellitus, síndrome nefrótico, tabaquismo, condiciones de inmunosupresión (VIH, inmunodeficiencias congénitas, malignidad, mieloma múltiple y corticoterapia,

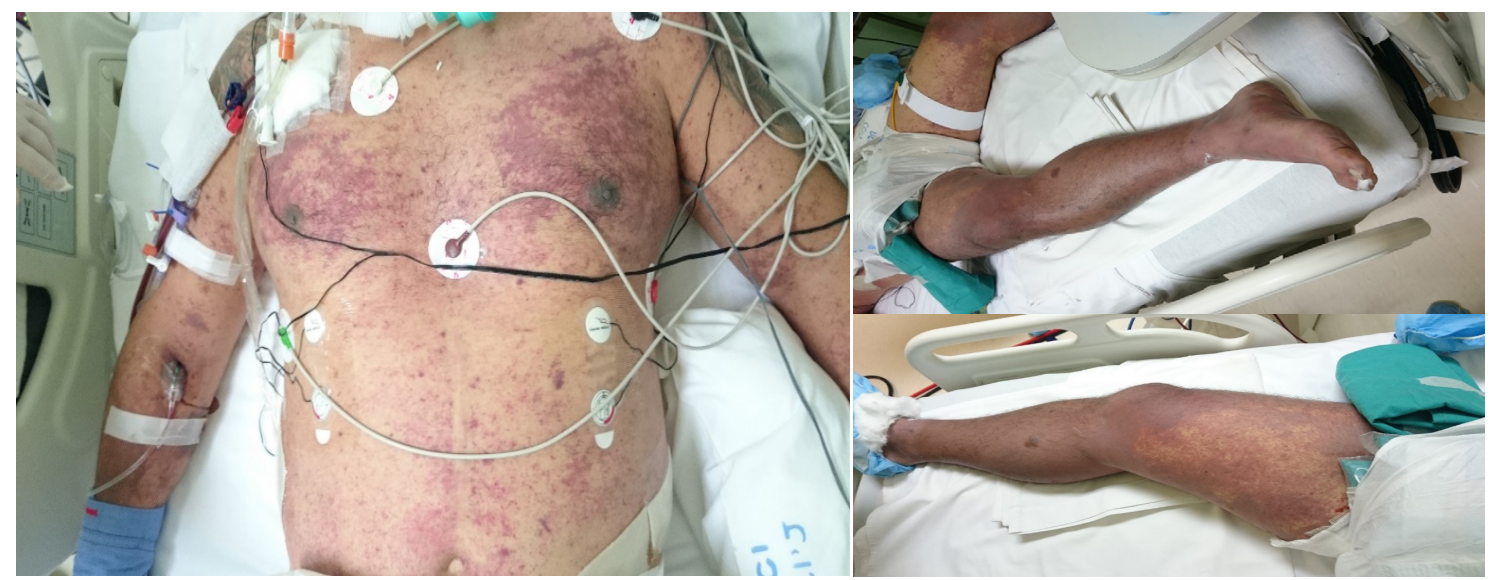

Figura I (A, B y C). Infección sobreaguda post esplenectomía por Streptoccus pneumoniae. Lesiones purpúricas confluentes en el tronco $y$ extremidades en tronco y extremidades. 
antineoplásicos). ${ }^{17}$ El tratamiento de esta condición está basado principalmente en la terapia antibiótica activa contra el Streptococcus pneumoniae y el soporte del paciente en función del sitio de infección implicado.

Sepsis fulminante postesplenectomía

La complicacion más grave en el paciente esplenectomizado es la OPSI. Esta se caracteriza por un pródromo inespecífico tipo gripal con fiebre, malestar general, mialgias, artralgias seguidos por un deterioro muy rápido hacia shock séptico fulminante, CID y muerte en las siguientes $24-48$ horas. ${ }^{18,19}$ El foco no suele ser obvio por tratarse de bacteriemia masiva, particularmente por gérmenes encapsulados con una alta virulencia. ${ }^{19}$ La OPSI es una condición grave y potencialmente fatal, lo que la convierte en una emergencia médica cuya identificación y tratamiento inmediatos marcan el pronóstico del paciente. ${ }^{11}$ El riesgo de OPSI es 50 veces mayor en esplenectomizados que en la población general. ${ }^{8}$ La prevalencia posterior a la cirugía puede alcanzar el $0,1 \%-0,5 \%,{ }^{20}$ Sin embargo, la mortalidad varía entre $50 \%-70 \%$, llegando a reducirse hasta $10 \%$ si se diagnostica tempranamente y se instaura terapia antibiótica oportuna y agresiva. ${ }^{19}$ A pesar de ello, la prevención de esta infección no siempre se realiza de forma oportuna $y$, como en este caso, los pacientes no reciben vacunación periesplenectomía.

La causa más frecuente de OPSI es $S$. pneumoniae, representando hasta el $50-90 \%$ de las infecciones y el $59 \%$ de las muertes, seguido por $H$. influenzae tipo b y $N$. meningitidis. ${ }^{9} 10$ Con menos frecuencia también están implicados Escherichia coli, Pseudomonas aeruginosa,

\begin{tabular}{|c|c|}
\hline Formas congénitas & Enfermedades autoinmunes \\
\hline $\begin{array}{ll}\text { - } & \text { Hiposplenia congénita aislada } \\
\text { - } & \text { Síndrome autoinmune de poliendocrinopatía - candidiasis - } \\
\text { distrofia ectodérmica (APECED) } \\
\text { - } \quad \text { Síndrome de hipoparatiroidismo } \\
\text { - } \quad \text { Sindrome de Stormorken }\end{array}$ & $\begin{array}{ll}- & \text { Lupus eritematoso sistémico } \\
- & \text { Artritis reumatoidea } \\
- & \text { Glomerulonefritis } \\
- & \text { Tiroiditis } \\
- & \text { Sarcoidosis } \\
- & \text { Poliarteritis nodosa } \\
- & \text { Síndrome de Sjögren }\end{array}$ \\
\hline Enfermedades gastrointestinales & Enfermedades infecciosas \\
\hline $\begin{array}{ll}\text { - } & \text { Enfermedad celiaca } \\
\text { - } & \text { Enfermedad inflamatoria intestinal } \\
\text { - } & \text { Enfermedad de Whipple }\end{array}$ & $\begin{array}{ll}- & \text { VIH-SIDA } \\
- & \text { Meningitis pneumocócica } \\
\text { - } & \text { Malaria }\end{array}$ \\
\hline Enfermedades hepáticas & Formas iatrogénicas \\
\hline $\begin{array}{ll}\text { - } & \text { Hepatitis crónica activa } \\
\text { - } & \text { Cirrosis biliar primaria } \\
\text { - } & \text { Hipertensión portal y cirrosis hepática } \\
\text { - } & \text { Hepatopatia alcohólica }\end{array}$ & $\begin{array}{ll}\text { - } & \text { Exposición a metildopa } \\
\text { - } & \text { Altas dosis de esteroides } \\
\text { - } & \text { Nutrición parenteral total } \\
\text { - } & \text { Irradiación esplénica } \\
\end{array}$ \\
\hline Enfermedades oncohematológicas & Alteraciones en la circulación esplénica \\
\hline $\begin{array}{ll}\text { - } & \text { Enfermedad mieloproliferativa crónica } \\
\text { - } & \text { Leucemia aguda } \\
\text { - } \quad \text { Transplante de médula ósea }\end{array}$ & $\begin{array}{ll}\text { - } & \text { Trombosis de la arteria esplénica } \\
\text { - } & \text { Trombosis de la vena esplénica } \\
\text { - } & \text { Trombosis de la arteria celiaca }\end{array}$ \\
\hline
\end{tabular}

\begin{tabular}{|l|l|}
\hline \multicolumn{2}{|l|}{ Tabla 2. Definición de términos } \\
$\begin{array}{l}\text { Enfermedad neumocócica invasiva o IPD (Invasive } \\
\text { Pneumococcal Disease) }{ }^{2,9-11}\end{array}$ & $\begin{array}{l}\text { Infección confirmada por aislamiento de Streptococcus pneumoniae de un sitio } \\
\text { normalmente estéril (ej. Sangre o LCR). }\end{array}$ \\
\hline IPE & $\begin{array}{l}\text { Infección sospechada o confirmada que requiere hospitalización y antibióticos } \\
\text { endovenosos. }\end{array}$ \\
\hline OPSI (Overwhelming Post-Splenectomy Infection) ${ }^{2,9-11}$ & $\begin{array}{l}\text { Es un subgrupo de IPE que progresa rápidamente a shock, coma y } \\
\text { muerte, requiere ingreso a unidad de cuidados intensivos (UCI), y causada } \\
\text { principalmente por gérmenes encapsulados. }\end{array}$ \\
\hline PF (Púrpura Fulminans) & $\begin{array}{l}\text { Desorden grave y potencialmente fatal, caracterizada por aparición rápida de } \\
\text { necrosis hemorrágica cutánea debido a trombosis vascular y CID. }\end{array}$ \\
\hline
\end{tabular}


Capnocytophaga canimorsus, Enterococcus sp, Bacteroides sp, and Bartonella sp. ${ }^{1}$ A pesar que el riesgo de OPSI es mayor en los primeros 3 años, sobre todo en el primer, durante el cual hasta el 17\% la desarrollan, llegando al 33\% a los 10 años, ${ }^{21}$ nuestro paciente la desarolló 20 años después. Esto es congruente con otros reportes que la han descrito hasta 20-40 años después de la esplenectomía. ${ }^{22,23}$ Condiciones que deprimen el sistema inmune podrían incrementar el riesgo de infección y muerte. A pesar de no tener factores de inmunosupresión, el desenlace de nuestro paciente fue rápidamente progresivo y fatal.

\section{Rol del bazo en infecciones por encapsulados}

¿Qué hace del bazo un órgano determinante para protegernos de OPSI e infecciones por encapsulados? Además de su función inmune (activación y proliferación de linfocitos T y B), el bazo se encarga de filtrar sangre (macrófagos eliminando virus, bacterias y celulas sanguíneas senescentes) y de la hematopoyesis. El 5\% del gasto cardíaco circula a través del bazo cada minuto, y aproximadamente el $10 \%$ de la sangre que ingresa a través de los capilares es vertida en los sinusoides venosos. El resto ingresa a una especie de sistema de circulación abierta en la pulpa roja, donde la sangre es forzada a entrar en los senos venosos. Puesto que las células sanguíneas deben atravesar los poros de los sinusoides venosos, el flujo sanguíneo sufre un enlentecimiento prominente, permitiendo que los macrófagos remuevan células defectuosas y microorganismos como bacterias y parásitos. $^{6} \mathrm{La}$ microestructura del bazo se divide en tres compartimentos, la pulpa blanca (PB), pulpa roja (PR) y la zona marginal (ZM). ${ }^{5}$ La pulpa roja está compuesta por sinusoides, capilares y cordones de Billroth, por donde fluyen y se filtran diversos componentes de la sangre como células rojas y blancas. La pulpa blanca está formada por la vaina linfoide periarteriolar que contiene linfocitos y está rodeada por folículos de células B en su capa más externa. La ZM es un área de tránsito, a través de la cual las células transitan hacia la pulpa blanca, y está compuesta principalmente por linfocitos B de memoria IgM que participan en la respuesta inmune innata y se encargan de producir una gran variedad de anticuerpos naturales. ${ }^{5}$ Los mecanismos moleculares que regulan este proceso no se conocen del todo bien. La gran mayoría de bacterias son fagocitadas directamente por macrófagos esplénicos mediante PRR (receptores de reconcimiento de patrones); sin embargo, otras requieren ser opsonizadas para su fagocitosis en el mismo bazo y en el hígado. ${ }^{24,25}$ Algunas bacterias son opsonizadas de forma deficiente debido a la presencia de una cápsula, en particular S. pneumoniae, cuya cápsula evita la unión del complemento o la interaccion del complemento ensamblado con los receptores del macrófago., 5 Afortunadamente, en la ZM existen dos grupos de macrófagos únicos del bazo bien diferenciados fenotípicamente, los cuales son capaces de fagocitar directamente bacterias encapsuladas a través de lectinas y sialoadesinas. Estos son, los macrófagos de la zona marginal y los macrófagos metalofílicos de la zona marginal. Los primeros expresan una lectina tipo $\mathrm{C}$ llamada SIGNR1 y el receptor scavenger tipo I, MARCO. SIGNR1 se une eficientemente a antígenos polisacáridos, como el lipoarabinomanano de M. tuberculosis. ${ }^{26}$ Además, esta lectina también participa en la captación y eliminación de antígenos polisacáridos de bacterias encapsuladas, ${ }^{27}$ y probablemente en la captación y eliminación de virus. ${ }^{28}$ MARCO, por su parte, se ha implicado en la captación de $E$. coli y $S$. aurerus. ${ }^{5}$ Por su parte, los macrófagos metalofílicos tienen alta expresión de SIGLEC1 (una sialoadesina), la cual además de unirse a residuos de ácido siálico de células del sistema inmune, también se une a aquellos resíduos de patógenos. ${ }^{30}$ Ésta sialoadesina ha demostrado mediar la captación y fagocitosis de $N$. meningitidis, y otros patógenos con residuos de ácido siálico en moléculas de superficie de membrana. ${ }^{5}$

Además de los mecanismos descritos hasta el momento, las bacterias encapsuladas requieren de anticuerpos naturales, los cuales son IgM pentaméricas, para facilitar su fagocitosis de manera directa o por depósito de complemento sobre la cápsula. Estos anticuerpos naturales son producidos por un tipo específico de células B presentes exclusivamente en el bazo, las células B de la zona marginal o células B IgM de memoria. ${ }^{1,5}$ Éstas tienen la capacidad única de producir una amplia variedad de anticuerpos naturales, incluyendo aquellos dirigidos contra $S$ pneumoniae, Neisseria meningitidis, y Haemophilus influenzae tipo b, iniciando así una respuesta inmune independiente de células $\mathrm{T}$, sea en respuesta a la infección natural o a la vacunación con antígenos polisacáridos. ${ }^{5}$ Se cree que su activación se produce por el desprendimiento de pequeñas moléculas de degradación de patógenos que han sido opsonizadas por el complemento. ${ }^{5,24}$ De esta forma, producen anticuerpos contra polisacáridos diferenciándose en células plasmáticas productoras de IgM específica, o convirtiéndose en potentes células presentadoras de antígeno que pueden migrar a la pulpa blanca y activar linfocitos T CD4 naive. ${ }^{31}$ Las células $\mathrm{B}$ de la zona marginal pueden diferenciarse fenotípicamnte por su alta expresion de IgM y CD1d (receptor del complemento C3d), y baja expresión de $\operatorname{IgD}$, a diferencia de las células $\mathrm{B}$ foliculares que tienen alta expresión de IgD y baja de IgM, y CD1d no detectable. ${ }^{32}$ De esta forma, los macrófagos de la zona marginal, macrófagos metalofílicos y las células B de la zona marginal juegan un rol protagónico en la protección de infecciones por gémenes encapsulados, de donde se desprende el rol fundamental del bazo contra estos microorganismos. La gran depleción de estas células $\mathrm{B}$ de memoria IgM y de macrófagos de la zona marginal es lo que determina la gran susceptibilidad a las infecciones por encapsulados en pacientes post esplenectomía o con hiposplenismo.

\section{Purpura fulminans}

La purpura fulminas, también conocida como púrpura gangrenosa, es una forma altamemte trombótica de 
coagulación intravascular diseminada, discapacitante y potencialmente fatal, caracterizada por aparición rápidamente progresiva de trombosis intravascular e infarto hemorrágico de la piel. ${ }^{33}$ Fue descrita por primera vez por Guelliot en 1884 en niños. ${ }^{32,33}$ La descripción clásica reportada hace poco más de 40 años por Spicer y Rau describía tres características principales: grandes lesiones purpúricas en la piel, fiebre e hipotensión. ${ }^{32}$ Fue descrita en escenarios tales como deficiencia de proteína $\mathrm{C}$, y más raramente de proteína $\mathrm{S}$; asociada a infecciones agudas como sepsis por bacterias gram negativas productoras de LPS (ej. Neisseria meningitidis); y aquella que ocurre 7-10 días después de una infección como resultado de aparición de autoanticuerpos con proteina $\mathrm{C}$ y proteína $\mathrm{S} .^{2}$ En nuestro caso, la causa final de muerte fue en el contexto de OPSI. Finalmente, los tres pilares para la prevención de OPSI son la educación del paciente, vacunación y profilaxis antibiótica. Una vez en curso el OPSI, el reconocimiento temprano y la terapia antibiótica oportuna y agresiva pueden reducir la mortalidad de un $70 \%$ a un $10 \% .{ }^{19}$

\section{REFERENCIAS BIBLIOGRÁFICAS}

1. Di Sabatino A, Carsetti R, Corazza GR. Post-splenectomy and hyposplenic states. The Lancet. 2011;378:86-97.

2. Chong J, Jones P, Spelman D, Leder K, Cheng AC. Overwhelming post-splenectomy sepsis in patients with asplenia and hyposplenia: A retrospective cohort study. Epidemiology and Infection. 2017 Jan $1 ; 145(2): 397-400$.

3. Kanhutu K, Jones P, Cheng AC, Grannell L, Best E, Spelman D. Spleen Australia guidelines for the prevention of sepsis in patients with asplenia and hyposplenism in Australia and New Zealand. Vol. 47, Internal Medicine Journal. Blackwell Publishing; 2017. p. 84855 .

4. Dendle C, Sundararajan V, Spelman T, Jolley D, Woolley I. Splenectomy sequelae: An analysis of infectious outcomes among adults in Victoria. Medical Journal of Australia. 2012 May;196(9):582-6.

5. Mebius RE, Kraal G. Structure and function of the spleen. Nature Reviews Immunology. 2005;5:606-16.

6. William BM, Corazza GR. Hyposplenism: A comprehensive review. Part I: Basic concepts and causes. Hematology. 2007;12:1-13.

7. Kraal G. Cells in the Marginal Zone of the Spleen. International Review of Cytology. 1992 Jan 1;132(C):31-74.

8. Hansen K, Singer DB. Asplenic-hyposplenic overwhelming sepsis: Postsplenectomy sepsis revisited. Pediatric and Developmental Pathology. 2001;4:105-21.

9. Tsigrelis C, Tleyjeh IM, Lahr BD, Nyre LM, Virk A, Baddour LM. Decreases in case-fatality and mortality rates for invasive pneumococcal disease in Olmsted County, Minnesota, during 19952007: a population-based study. Clinical infectious diseases. 2008 Dec 1;47(11):1367-71

10. Holdsworth RJ, Cuschieri A, Irving AD. Postsplenectomy sepsis and its mortality rate: Actual versus perceived risks. British Journal of Surgery. 1991 Sep 1;78(9):1031-8.

11. Sinwar PD. Overwhelming post splenectomy infection syndrome review study. International Journal of Surgery (London, England). 2014 Dec 1;12(12):1314-6.

12. Morgan TL, Tomich EB. Overwhelming post-splenectomy infection (OPSI): a case report and review of the literature. The Journal of Emergency Medicine. 2012 Oct;43(4):758-63.

13. Quinti I, Paganelli R. Asplenia. In: Stiehm's Immune Deficiencies. Elsevier Inc.; 2014. p. 835-44.

14. Surveillance Manual | Pneumococcal | Vaccine Preventable Diseases | CDC [Internet]. [cited 2020 Mar 19]. URL disponible en: https:// www.cdc.gov/vaccines/pubs/surv-manual/chpt11-pneumo.html

15. Taylor SN, Sanders CV. Unusual manifestations of invasive pneumococcal infection. The American Journal of Medicine. 1999 Jul 26;107(1A):12S-27S.

16. Darmstadt GL. Acute infectious purpura fulminans: Pathogenesis and medical management. Pediatric Dermatology. 1998;15:169-83.

17. Centers for Disease Control and Prevention (CDC). Use of 13-valent pneumococcal conjugate vaccine and 23-valent pneumococcal polysaccharide vaccine for adults with immunocompromising conditions: recommendations of the Advisory Committee on Immunization Practices (ACIP). MMWR. 2012 Oct 12;61(40):816-9.

18. Tahir F, Ahmed J, Malik F. Post-splenectomy sepsis: A review of the literature. Cureus. 2020;12(2): e6898. DOI 10.7759/cureus.

19. Brigden ML. Overwhelming postsplenectomy infection still a problem. The Western Journal of Medicine. 1992 Oct;157(4):440-3.

20. Sarangi J, Coleby M, Trivella M, Reilly S. Prevention of post splenectomy sepsis: A population- based approach. Journal of Public Health (United Kingdom). 1997 Jun;19(2):208-12.

21. Schwartz PE, Sterioff S, Mucha P, Melton LJ, Offord KP. Postsplenectomy sepsis and mortality in adults. JAMA. 1982 Nov 12;248(18):2279-83.

22. Dennis Robinette C, Fraumeni JF. Splenectomy and subsequent mortality in veterans of the 1939-45 war. The Lancet. 1977;310:127-9.

23. KING H, SHUMACKER HB. Splenic studies. I. Susceptibility to infection after splenectomy performed in infancy. Annals of Surgery [Internet]. 1952 Aug [cited 2020 Mar 23];136(2):239-42. URL disponible en: http://www.ncbi.nlm.nih.gov/pubmed/14953147

24. Kang Y-S, Yamazaki S, Iyoda T, Pack M, Bruening SA, Kim JY, et al. SIGN-R1, a novel C-type lectin expressed by marginal zone macrophages in spleen, mediates uptake of the polysaccharide dextran. International immunology. 2003 Feb;15(2):177-86.

25. Koppel EA, Ludwig IS, Hernandez MS, Lowary TL, Gadikota RR, Tuzikov AB, et al. Identification of the mycobacterial carbohydrate structure that binds the C-type lectins DC-SIGN, L-SIGN and SIGNR1. Immunobiology. 2004;209(1-2):117-27.

26. Geijtenbeek TBH, Groot PC, Nolte MA, van Vliet SJ, GangaramPanday ST, van Duijnhoven GCF, et al. Marginal zone macrophages express a murine homologue of DC-SIGN that captures blood-borne antigens in vivo. Blood. 2002 Oct 15;100(8):2908-16.

27. Oehen S, Odermatt B, Karrer U, Hengartner H, Zinkernagel R, López-Macías C. Marginal zone macrophages and immune responses against viruses. Journal of immunology (Baltimore, Md. : 1950). 2002 Aug 1;169(3):1453-8.

28. Elomaa O, Sankala M, Pikkarainen T, Bergmann U, Tuuttila A, Raatikainen-Ahokas A, et al. Structure of the human macrophage MARCO receptor and characterization of its bacteria-binding region. Journal of Biological Chemistry. 1998 Feb 20;273(8):4530-8.

29. Betrosian AP, Berlet T, Agarwal B. Purpura fulminans in sepsis. American Journal of the Medical Sciences. 2006;332:339-45.

30. Jones C, Virji M, Crocker PR. Recognition of sialylated meningococcal lipopolysaccharide by siglecs expressed on myeloid cells leads to enhanced bacterial uptake. Molecular microbiology. 2003 Sep;49(5):1213-25.

31. Martin F, Kearney JF. Marginal-zone B cells. Nature reviews Immunology. 2002 May;2(5):323-35.

32. Colling ME, Bendapudi PK. Purpura Fulminans: Mechanism and Management of Dysregulated Hemostasis. Vol. 32, Transfusion Medicine Reviews. W.B. Saunders; 2018. p. 69-76.

33. Spicer TE, Rau JM. Purpura fulminans. The American Journal of Medicine. 1976 Oct 1;61(4):566-71.

\section{CORRESPONDENCIA:}

Luis Novoa Millones

luisnm8@gmail.com

Fecha de recepción: 28-09-2020.

Fecha de aceptación: I6-II-2020. 